\title{
Diagnosis of a Gangrenous, Strangulated \& Incarcerated Inguinal Hernia by Identifying C-Shaped Small Bowel Loops and the Serrated Beak Appearance
}

\author{
Lin $\mathrm{MP}^{1,2,3}$, Chen $\mathrm{YL}^{2}$ and Ginger HF Shu ${ }^{1 *}$ \\ ${ }^{1}$ Department of Radiology, Chi-Mei Foundation Medical Center, Tainan City, ROC, Taiwan \\ ${ }^{2}$ Cheng Shiu University, Kaohsiung City, ROC, Taiwan \\ ${ }^{3}$ Chung Hwa University of Medical Technology, Tainan City, ROC, Taiwan
}

*Corresponding author: Ginger HF Shu, MD, MPH, Department of Radiology, Chi-Mei Foundation Medical Center, Tainan City, ROC, Taiwan, Tel: 886.6.726.3333, E-mail: mariposa@seed.net.tw

Citation: Lin MP, Yen-Li Chen YL, Ginger HF Shu (2018) Diagnosis of a Gangrenous, Strangulated \& Incarcerated Inguinal Hernia by Identifying C-Shaped Small Bowel Loops and the Serrated Beak Appearance. SAJ Case Rep 5: 407

Article history: Received: 01 November 2017, Accepted: 05 October 2018, Published: 09 October 2018

\begin{abstract}
A gangrenous, strangulated, and incarcerated inguinal hernia is a rare cause of intestinal obstruction, accounting for only $0.29 \%$ to $2.9 \%$ of all causes of inguinal hernias. In this case report, we present a case of gangrenous, strangulated, and incarcerated inguinal hernia who had undergone imaging study with computed tomography. The patient is an 81-year-old elderly female with a painful, protruding and non-reducible mass over the right inguinal region for 10 days. Laboratory studies revealed leukocytosis with a white blood cell count of $19.8 \times 10^{3} / \mathrm{uL}$ (normal values at our institution are $3.4-4.9 \times 10^{3} / \mathrm{uL}$ ). The C-reactive protein level of the patient was elevated at 166.3 $\mathrm{mg} / \mathrm{dl}$ (>6 mg/dl). A computed tomography (CT) imaging study was performed for further clinical assessment.

CT revealed C-shaped bowel loops characterized with a "serrated beak" appearance at the transition point suggesting a strangulated right inguinal hernia. Laparotomy confirmed the presence of a strangulated and incarcerated right inguinal hernia. CT findings of C-shaped bowel loops with a serrated beak appearance are important in achieving a correct diagnosis and providing helpful information pertaining to clinical management and optimal treatment planning.
\end{abstract}

Keywords: Computed Tomography; C-shaped bowel loops; Gangrene; Incarcerated; Inguinal Hernia; Serrated beak appearance; Strangulated

\section{Introduction}

An inguinal hernia is an abnormal protrusion of intra-abdominal structures through a fascial defect in the groin. When a bowel segment inside the hernia sac becomes obstructed, interference of circulation may ensue. Strangulation of the inguinal hernia is present in the patient in our case report, a condition in which gangrene of the contents in the hernia sac has occurred. A strangulated inguinal hernia is a life-threatening condition that requires urgent surgical intervention. Historically, prominent adhesive bands are a well-established cause of intestinal obstruction. Documentations of hernias can be traced back to the time of Hippocrates, and the catastrophic consequences incarcerated hernias have been known since time immemorial. Celsus had referred to a number of interesting historical incidences and was the first to detail surgical techniques for the correction of hernias. Needless to say, these measures, involving various techniques of cauterization or the use of caustic substances are extremely primitive compared with modern day techniques [1-4].

\section{Case Report}

The patient in our case report is an 81-year-old elderly female suffering from a painful, protruding, and non-reducible mass over the right inguinal region for 10 days. The patient had never visited the emergency department prior to her present condition. Upon admission, her vital signs were stable, and laboratory studies revealed leukocytosis with a white blood cell count of $19.8 \times 10^{3} / \mathrm{uL}$ (normal values at our institution are $3.4-4.9 \times 10^{3} / \mathrm{uL}$ ). The C-reactive protein level of the patient was elevated at $166.3 \mathrm{mg} / \mathrm{dl}(>6$ $\mathrm{mg} / \mathrm{dl}$ ). Physical examination revealed a tense, bulging, painful and erythematous mass $12 \mathrm{~cm}$ in size over the right inguinal region. An incarcerated inguinal hernia was suspected, and computed tomography (CT) revealed C-shaped bowel loops with a "serrated beak" appearance at the transition point suggesting strangulation of an incarcerated right inguinal hernia (Figure 1). Laparotomy confirmed the diagnosis of a gangrenous, strangulated and incarcerated hernia. 

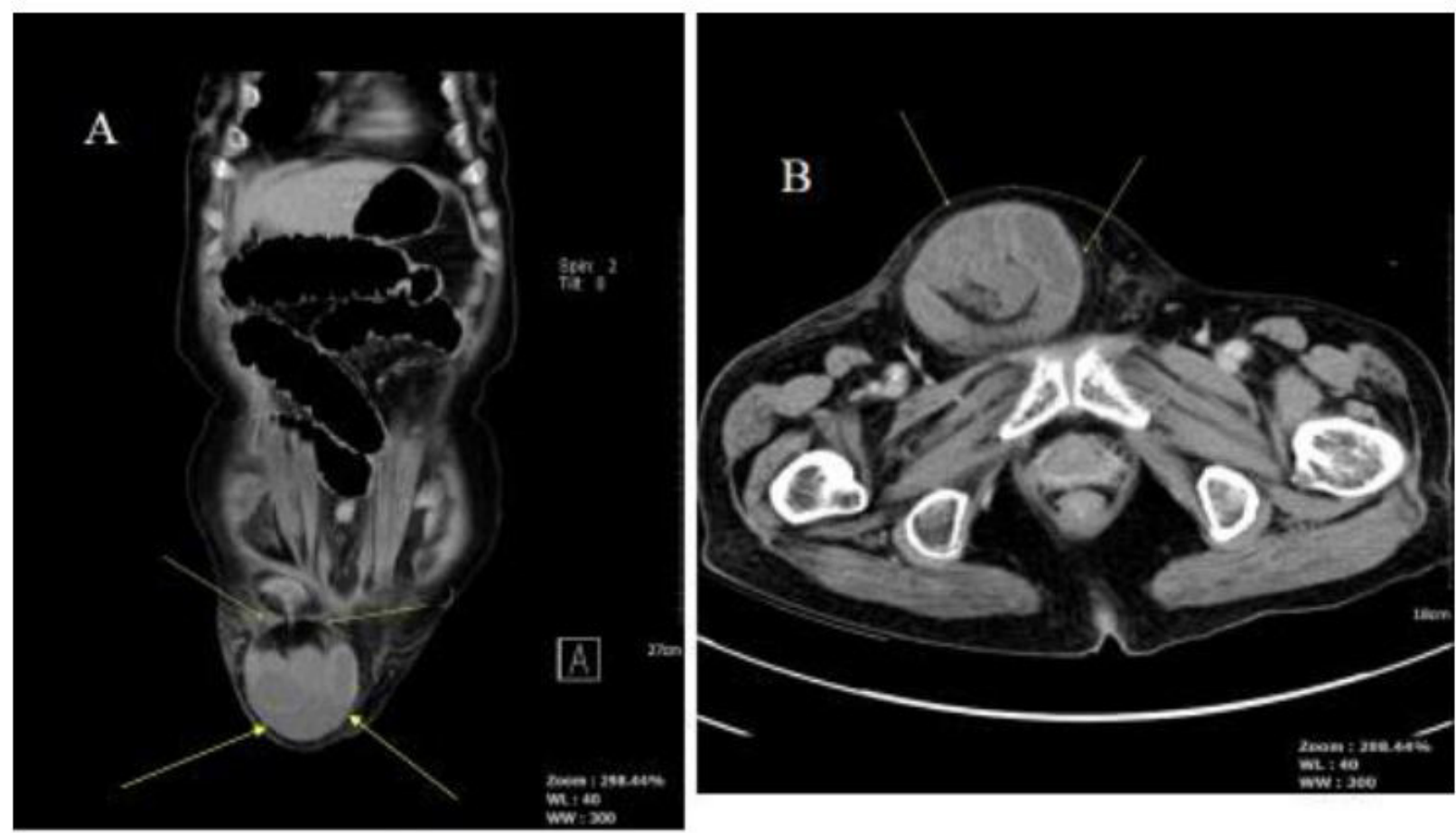

Figure 1: Strangulated hernia in an 81-year-old woman

(a) Coronal; (b) Axial contrast-enhanced reformatted CT image of the abdomen shows a strangulated inguinal

hernia (arrows) causing small bowel obstruction (arrows). A herniated bowel loop with a serrated beak and

C-shaped configuration, discrete mesenteric engorgement within the hernia sac

\section{Discussion}

Hernias are protrusions of normal anatomic structures through fascial and muscular layers containing them. An inguinal hernia occurs when various layers of the abdominal wall fail to contain enclosed viscera. The most common clinical presentation in nearly all patients is the appearance of a lump in the groin region, sometimes accompanied by pain or a dragging sensation. Hernias may initially resolve by themselves only to reappear with coughing, straining or standing. Complications may eventually develop, such as irreducibility and obstruction with or without strangulation, which could quickly turn an easily treatable condition into a lifethreatening emergency. Identification of risk factors may be helpful in predicting complications in certain patient groups. Early admission and prompt surgery of high-risk patients may significantly reduce mortality and morbidity [5].

Inguinal hernias may become irreducible and result in intestinal obstruction, but strangulation is, by far, the complication of greatest concern and requires urgent surgical intervention. Strangulation occurs when circulation is compromised. Venous congestion results from partial blood supply interruption; gangrene occurs in cases of complete blood supply occlusion.

Although much less frequently encountered than inguinal hernias, femoral hernias are much more prone to become strangulated. The incidence of strangulation in inguinal hernias is up to 5\% [6]. Risk factors predicting complications in the adult population are advanced age, short duration of hernia, femoral type hernias, and co-existing medical illnesses [5-7].

Exact precipitating causes of bowel obstruction and strangulation are unclear, but coughing or sneezing may force intra-abdominal contents into the hernia sac, which are then pinched at the neck of the sac by the deep ring. The contents within the hernia sac become swollen due to venous congestion, which in turn causes impair of arterial circulation and ensuing gangrene within 4-6 hours. If left untreated, gut perforation may occur, leading to localized sepsis followed by generalized septicemia.

Large, long-standing inguinal hernias are more commonly the sliding variety of hernias. Neglected cases, such as patients in nursing homes, hospices and psychiatric wards, may develop necrosis of the overlying skin. Sometimes, the sac may even slough off, resulting in intestinal protrusion through the defect. In our patient, most of the small bowel was protruding out of the sac and was observed lying in front of the upper thigh. Gangrene of the bowel was due to both arterial and venous occlusion at the level of the deep ring. This patient was elderly and living alone, and systemic sepsis was observed by the time she was brought to medical attention. The most common cause of intestinal perforation is bowel necrosis at the ante-mesenteric border due to impairment of vascular perfusion. In cases of large inguinal hernias, even direct trauma may cause bowel perforation [8].

Standard laparotomy is the recommended treatment of a strangulated inguinal hernia because adequate gut resection after identifying the vascular structures is only possible through abdominal incision [9]. Intestinal resection and anastomosis are performed through a midline celiotomy, and Bassini's repair is undertaken to treat the hernia. After debridement, the scrotal wound is packed in male patients. 
After undergoing surgical intervention, the patient in our case report made a slow but steady post-operative recovery. The extent and severity of her disease is rarely encountered in this modern of age of sophisticated surgery and anesthesia. Although conservative treatment may be instituted in frail, elderly patients who are poor surgical candidates, the potential of serious complications, including strangulation and gangrene, makes it such an undesirable option that some countries in the world have adopted the policy of operating on all hernias [10]. Elective hernia repair in frail and elderly patients, even those on continuous ambulatory dialysis, has shown to be associated with lower mortality as compared to opting for conservative treatment [11].

\section{Conclusion}

Gangrene resulting from strangulation refers to ischemia caused by compromised vascular perfusion. It usually occurs when a hernia causes obstruction of the afferent and efferent intestinal loops, creating a closed loop within the herniated bowel. Findings on CT scans include closed-loop obstruction and evidence of ischemia as well as dilated, fluid-filled, U- or C-shaped loop of bowel entrapped within the hernia sac [12]. Imaging findings indicating ischemia include bowel wall thickening, abnormal mural hypo- or hyper attenuation and enhancement, and engorgement of the mesenteric vessels. Afferent and efferent limbs of the involved bowel may exhibit a "serrated beak" appearance at the transition point (1).CT findings in inguinal hernias are important in establishing an accurate diagnosis and providing helpful information pertaining to clinical management and optimal treatment planning.

\section{References}

1. Lichtenstein IL (1986) Hernia repair without disability. (2 ${ }^{\text {nd }}$ edn) St Louis, Mo: ishlyaku Euroamerica Inc.

2. Rutkow IM (1997) Surgical operations in the United States; then (1983) and now (1994). Arch Surg 132: 983-90.

3. Kingsworth A, Bennet DH, Hernias (2000) Umbilicus: abdominal wall. In: Russell RCG, Williams NS, Bulstrode CJK. Bailey and Love Short Practice of Surgery $\left(23^{\text {rd }}\right.$ edn) London.

4. Giles GR, Halasz NA (1995) The Abdominal Wall and Hernias. In: Cuschieri A, Giles GR, Moossa AR. Essential Surgical Practice $3^{\text {rd }}$ edn. London: Butterworth-Heinernann: 1445.

5. Rai S, Chandre SS, Smile SR (1998) A study of the risk of strangulation and obstruction in groin hernias. Aust NZJ Surg 68: 650-4.

6. Uppot RN, Ghey VK, Gupta R (2000) Intestinal perforation from blunt trauma to inguinal hernia. Am J Roentgenol; 174:1538.

7. Chaloner EJ, Deineuvilie J (1997) External strangulation of the small bowel following injury to inguino-scrotal hernia. Injury 28 : 69-70.

8. Horban VR, Merliuk VO, Arsenink VV (1998) Strangulated intramural ingtiinal hernia. Kiln Khir 3: 53.

9. Devlin HB (1983) Inguinal hernia in adults. In: Dudley H (ed) Rob and Smith operative surgery $4^{\text {th }}$ edn. London: Butterworth-Heinemann: 465.

10. Millat B (1997) Treatment of inguinal hernia. Rer Prat: 47: 268-72.

11. Morris-Stiff GJ, Bowrey DJ, Jurewicz WA (1998) Management of inguinal hernia in patients on continuous ambulatory peritoneal dialysis: an audit current UK practice.

12. Aguirre DA, Santosa AC, Casola G, Sirlin CB (2005) Abdominal wall hernias: imaging features, complications, and diagnostic pitfalls at multi-detector row CT. Radio Graphics 25: 1501-20. 